\title{
FRUTA DO CONDE E SAÚDE (Annona squamosa, L.): UMA BREVE REVISÃo
}

\author{
SUGAR-APPLE AND HEALTH (Annona squamosa, L.): A BRIEF REVIEW
}

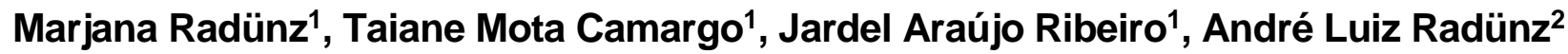

1 - Programa de Pós-Graduação em Ciência e Tecnologia de Alimentos - Universidade Federal de Pelotas, RS 2 - Docente do Departamento de Agronomia - Universidade Federal da Fronteira Sul - Campus Chapecó, SC Autor correspondente: marjanaradunz@gmail.com

\section{RESUMO:}

A busca por uma alimentação saudável que ocasione a promoção da saúde vêm aumentando nos últimos anos, e, neste sentido, as frutas e hortaliças recebem destaque, já que seu consumo está associado com a menor incidência de diversas doenças, tais como diabetes mellitus tipo II, doenças cardiovasculares e cânceres. Dentre as frutas que possuem estes benefícios está a fruta do conde (Annona squamosa, L.), que é produzida principalmente na região nordeste do Brasil. A mesma é rica em compostos fenólicos, terpenos, e as acetogeninas, ácidos graxos de cadeia longa exclusivas da família Annonaceous. Estes compostos são responsáveis por diversas atividades atribuídas à fruta, como atividade antioxidante, antimicrobiana, anticarcinogênica, anti-hipertensiva, antidiabética, imunossupressora, além de amenizar os sintomas do hipotireoidismo, comprovando, assim, que o seu consumo é extremamente recomendável e benéfico ao organismo humano.

Palavras chave: antitumoral; antidiabetogênico; compostos fenólicos; atividade antioxidante.

\section{ABSTRACT:}

The search for a healthy diet that occasion health promotion has been increasing in recent years, and in this sense, fruits and vegetables are highlighted, since their consumption is associated with the lower incidence of various diseases, such as type II diabetes mellitus, cardiovascular diseases and cancers. Among the fruits that have these benefits is the sugarapple (Annona squamosa, L.), which is produced mainly in the northeastern region of Brazil. It is rich in phenolic compounds, terpenes, and the acetogenins, long chain fatty acids exclusive to the Annonaceous family. These compounds are responsible for several activities attributed to the fruit, such as antioxidant, antimicrobial, anticarcinogenic, antihypertensive, antidiabetic, immunosuppressive activity, as well as mitigating the symptoms of hypothyroidism, thus proving that its consumption is extremely recommendable and beneficial to the body human.

Keywords: antitumor, antidiabetogenic. phenolic coumpounds, antioxidant activity.

\section{INTRODUÇÃO}

A fruta do conde (Annona squamosa, L.), também conhecida como pinha, ata ou araticum é uma anonácea pertencente à família das Annonaceae, originária da llha de Trindade e das Antilhas. O seu cultivo no Brasil iniciou em 1926 na Bahia, posteriormente se estendendo para outros estados do Nordeste como Alagoas, Ceará, Paraíba, 
Pernambuco, e alguns estados do Sudeste como Minas Gerais, Rio de Janeiro e São Paulo (MEDINA et al., 2015). Sua produção na região nordeste pode ocorrer durante todo o ano, desde que apresente condições de irrigação, uso intensivo de adubos e defensivos agrícolas, além de grande mão de obra (CARLSON et al., 2007).

Quanto ao clima ideal de cultivo, a árvore se adapta a condições do litoral e do semiárido, por não tolerar temperaturas muitas baixas, sendo a faixa de temperatura mínima de 10 a $20^{\circ} \mathrm{C}$ e temperaturas máximas de 22 a $28^{\circ} \mathrm{C}$. A definição do momento da colheita é de extrema importância, pois é na fase final de seu desenvolvimento que a fruta forma polpa de enchimento mais saborosa. Essa maturação se inicia com o afastamento dos carpelos e promove mudanças na cor, sabor, aroma e textura (VIANA, 2005).

$\mathrm{A}$ árvore possui porte pequeno, de 4 a $6 \mathrm{~m}$ de altura, muito ramificada, com folhas lanceoladas de 6 a $7 \mathrm{~cm}$ de comprimento, decíduas e de coloração verde brilhante na parte superior, e verde azulada na parte inferior. As sépalas se apresentam em três e são triangulares, medindo 2 a $3 \mathrm{~cm}$ de comprimento. As três pétalas externas são lanceoladas e grossas com $1,5 \mathrm{~cm}$ de comprimento, de coloração amarelo verdosas por fora, e amareladas com uma mancha roxa na base isoladas ou na forma de cachos contendo de 2 a 4 flores. O seu fruto é um sincarpo arredondado de 5 a $10 \mathrm{~cm}$ de diâmetro, com peso entre 150 a $650 \mathrm{~g}$, formado por carpelos proeminentes e cobertos por saliências achatadas em forma de tubérculos, com coloração verde, polpa de cor branca translúcida ou creme, doce, rica em sais minerais e vitaminas, com cerca de 60 sementes de cor preta em cada fruto (EMBRAPA AGROINDÚSTRIA TROPICAL, 1998).

Segundo Alves et al. (2000), sua composição média é constituída por $39,16 \%$ de casca, $11,03 \%$ de sementes, $48,13 \%$ de polpa, sendo que a fruta e amadurece entre 100 a 120 dias após o florescimento, com mudança na coloração da casca que passa de verdeamarelada para creme-rosada no período de dezembro a julho, tendo seu maior pico em fevereiro e março. É relatado que esta planta contém carboidratos, proteínas, lipídeos e compostos fenólicos. Os carboidratos constituem entre 18,2 e $26,2 \%$, sendo destes $83 \%$ dos açúcares solúveis totais constituídos por açúcares redutores. Apresentam ainda teores altos de amido $(0,87 \%)$ e de pectina $(0,66 \%)$, ao final do processo de maturação, dificultando o processamento do suco. As sementes geralmente são descartadas, entretanto estas apresentam em sua composição substâncias chamadas acetogeninas, que apresentam diversas atividades biológicas, entre elas o potencial antioxidante e antimicrobiano (TU et al., 2016).

Baseado no exposto, o objetivo do presente trabalho é realizar uma revisão sobre 
os potenciais benefícios da fruta do conde para a saúde humana.

\section{METODOLOGIA}

O presente estudo foi do tipo exploratório-descritivo por meio de pesquisas bibliográficas oriundas de publicações e resultados de pesquisas específicas sobre a temática dos benefícios da fruta do conde a saúde humana. As bases de dados utilizadas foram Science Direct, Scielo, PubMed e Lilacs utilizando os seguintes descritores dos últimos 20 anos: fruta do conde, Annona squamosa, Annona squamosa e saúde, acetogeninas e acetogenins. Ao final da busca foram encontrados 2903 artigos, destes foram selecionados artigos que que apresentavam relevância sobre a temática abordada, totalizando 25 artigos.

\subsection{BENEFÍCIOS DA FRUTA DO CONDE (Annona squamosa, L.)}

Os benefícios a saúde atribuídos a fruta da Annona squamosa, L. são numerosos, no entanto, esta planta parece possuir potentes princípios bioativos na maioria de suas partes (frutas, sementes e folhas). Os vários constituintes químicos isolados das folhas, caules e raízes incluem anonaina, aporfina, corilelina, isocordina, norcorydina e glaucina que apresentam potencial antimicrobiano, antioxidante, antidiabetogênico e antitumoral (NAIR; AGRAWAL, 2017). No entanto, a presença de acetogeninas é um dos compostos que diferencia a fruta do conde outras espécies frutíferas. As acetogeninas são ácidos graxos de cadeia muito longa exclusivas da família Annonaceae, conferindo a esta planta atividade anticancerígena (NAIR; AGRAWAL, 2017). Estes compostos são obtidos através da via do ácido acético derivados de ácidos graxos de cadeia longa, com média de 35 a 39 carbonos. Apresentam uma cadeia alifática longa com hidroxila, acetila, carbonila e um anel $\gamma$-lactona terminal, podendo conter ainda de 1 a 3 anéis tetraidrofurânico ou anel tetraidropirânico. Existem sete classificações de acetogeninas: annona, asimina, disepalum, goniothalamus, rollinia, uvária e xylopia. Destes, segundo Alali et al., (1999) a annona é a que se encontra presente na fruta do conde (Annona squamosa, L.), já Cordeiro et al., (2000) identificou alem da anonina ou anonacina, a asimicina, bulatacina, bulatacinona e escuamocina.

Os estudos in vitro $\mathrm{e}$ in vivo demonstram propriedades anticancerígenas consideráveis e propriedades anti-hipertensivas. Algumas das propriedades obtidas através 
do uso de diferentes partes desta planta são demonstradas abaixo.

\subsection{Folhas}

No estudo realizado por Brito et al. (2008), foi possível a identificação a partir do extrato etanólico das folhas da fruta do conde (Annona squamosa, L.) a presença de alcaloides, flavanoides, flavononas, triterpenoides, esteroides, flavonas, flavonois, xantonas, saponinas, taninos e resinas. Os flavonoides e alguns fenólicos seriam os responsáveis pela atividade antioxidante da fruta do conde (Annona squamosa, L.). As propriedades hipoglicemiantes e antidiabéticas estudadas por Gupta et al., (2005) também a partir de extrato etanólico das folhas da Annona squamosa, L. apontaram potencial utilidade do extrato para tratar diabetes mellitus, ajudando a diminuir administração de insulina (NAIR; AGRAWAL, 2017). Outra ação benéfica das folhas de Annona squamosa, L. foi estudada Sunanda e Anand (2003), sugerindo que o extrato aquoso pode melhorar os sintomas de hipertireoidismo que é frequentemente considerado como um fator causador do diabetes (SHIRWAIKAR et al., 2004).

\subsection{Polpa}

Além dos nutrientes como carboidratos, proteínas e vitaminas, a fruta do conde é conhecida por conter metabólitos secundários como compostos fenólicos, alcaloides, saponinas (BASKARAN et al., 2016). Compostos fenólicos são metabólitos secundários que são amplamente encontrados em alimentos derivados de plantas. Estes compostos têm recebido atenção considerável como fatores potencialmente protetores contra o câncer e doenças cardíacas, principalmente por causa de suas propriedades antioxidantes. Utilizando UPLC-ESI-MS, Baskaran et al. (2016), encontraram cerca de 16 ácidos fenólicos livres, 15 ligados e 13 esterificados, respectivamente, na fruta do conde, indicando que o consumo desta fruta pode contribuir para a ingestão de polifenóis na dieta, e, por consequência, promover efeitos benéficos à saúde. Um dos benefícios é a atividade antitumoral estudada por Chen et al. (2017) através de diferentes extratos do pericarpo da Annona squamosa, L. Através de testes in vitro, esses autores verificaram efeito próapoptose para alguns tipos de células tumorais usando extrato obtido partir do acetato de etila. As propriedades anticancerígenas da fruta do conde parecem ser principalmente devido a uma classe de compostos chamados acetogeninas (NAIR; AGRAWAL, 2017). As 
acetogeninas foram testadas in vitro contra 60 tipos de células cancerígenas, incluindo mama, próstata e cólon. Em comparação com o paclitaxel, um fármaco anticancerígeno padrão, a bullatacina, uma acetogenina, foi 300 vezes mais potente, mesmo no sistema de teste in vivo (NAIR; AGRAWAL, 2017). Equivalente a ação das folhas, a polpa da fruta do conde também apresenta ação antiglicêmica, pois a pinha parece desenvolver o mesmo papel da insulina, estimulando a sua produção e aumentando a captação de glicose pelos músculos, o que leva à estabilização das concentrações de açúcar no sangue (NAIR; AGRAWAL, 2017). A polpa também apresenta efeito antitumoral contra tumores hepáticos (CHEN et al., 2017), alta atividade antioxidante e antidiabetogênica (NANDHAKUMAR; INDUMATHI, 2013; MOHD et al., 2009, PARDHASARADHI et al., 2005; PARDHASARADHI et al., 2004). Além disto, apresenta capacidade imunoreguladora (TU et al., 2016), e atua inibindo a atividade da $\alpha$-glicosidase (REN et al., 2017).

\subsection{Sementes}

Há estudos indicando que as sementes de fruta do conde apresentam efeitos antioxidantes frente aos radicais ácido 2,2'-azinobis-3-etilbenzotiazolina-6-sulfonico (ABTS), poder de redução de ferro (FRAP, 2,2-difenil-1- picrilhidrazil, (DPPH), hidroxila, óxido nítrico e superóxido (BIBA et al., 2017). Além disto, apresenta atividade antidiabética, hepatoprotetora, citotóxica e antitumoral (PANDEY \& BARVE, 2011). As sementes apresentam compostos bioativos como flavonoides, cumarinas, alcaloides e terpenoides (BIBA et al., 2013). Considera-se como compostos com atividade antimicrobiana na fruta do conde as acetogeninas e os terpenos.

A fruta do conde apresenta na sua composição química compostos com atividade citotóxica, antitumoral, pesticida, vermicida, antimicrobiana, imunossupressora, antiemética, inibidora do apetite e antimalárica (BRITO et al., 2008).

Terpenos são hidrocarbonetos múltiplos do isopreno, sua biossíntese ocorre a partir da combinação de duas moléculas de ácido acético para formar ácido mevalônico. Na polpa e nas sementes foram identificados compostos monoterpenos como a-pineno, sabineno, limoneno e (E) - $\beta$-ocimeno e também compostos sesquiterpenos, como o espatulenol, germacreno e o biciclogermacreno (ANDRADE et al., 2001). As sementes apresentam elevada concentração de óleos voláteis, onde foram identificados e isolados 6 componentes desses óleos com comprovada atividade antimicrobiana (RAHMAN et al., 2005; CHAVAN et. al, 2006). 


\section{CONCLUSÃO}

Conclui-se que o consumo da fruta do conde ocasiona inúmeros benefícios à saúde, já que as folhas, polpa e sementes apresentam em sua composição acetogeninas, terpenos e compostos fenólicos, que possuem diversas atividades benéficas ao organismo humano, tais como atividade antioxidante auxiliando na eliminação de radicais livres, anticarcinogênica frente a tumores de mama, próstata e cólon, antidiabética, antihipertensiva, dentre outras atividades importantes na promoção da saúde.

\section{REFERÊNCIAS}

ALALI, F.Q.; LUI, X.; MCLAUGHLIN, J.L. Annonaceous Acetogenins: recent progress. Journal of Natural Products, v. 62, p. 504-540, 1999.

ALVES, C.Q.; DAVID, J.M.; DAVID, J.P.; BAHIA, M.V.; AGUIAR, R.M. Métodos para determinação de atividade antioxidante in vitro em substratos orgânicos. Química Nova, v. 33, n. 10, p. 2202-2210, 2000.

ANDRADE, E.H.A; ZOGHBI, M.G.B; MAIA, J.G.S. Chemical characterization of the fruit of Annona squamosa L. occurring in the Amazon. Journal of Food Composition and Analysis, v. 14, p. 227-232, 2001.

BASKARAN, R; PULLENCHERI, D; SOMASUNDARAM, R. Characterization of free, esterified and bound phenolics in custard apple (Annona squamosa $L$ ) fruit pulp by UPLCESI-MS/MS. Food Research International, v. 82, p. 121-127, 2016.

BIBA, V.; AKHIL, B. S.; REMANI, P.; SUJATHAN, K. Free Radical Scavenging Properties of Annona squamosa, L. Asian Pacific Journal of Cancer Prevention, v.18, p. 2725-2731, 2017.

BIBA, V.S.; LAKSHMI, S.; DHANYA, G.S.; REMANI. P. Phytochemical analysis of Annona squamosa seed extracts. International Research Journal of Pharmaceutical and Applied Sciences, v. 3, p. 29-31, 2013.

BRITO, H.O.; NORONHA, E.P.; FRANÇA, L.M.; BRITO, L.M.O.; PRADO, M.S.A. Análise 
da composição fitoquímica do extrato etanólico das folhas da Annona squamosa (ATA). Revista Brasileira de Farmacologia, v. 89, n. 3, p. 180-184, 2008.

CARLSON, J. G.S, CHAVES, M.A.; SÃO JOSÉ, A.R.; REBOUÇAS, T.N.H.; ALVES, J.F.T. A influência da cobertura morta sobre características físicas e químicas dos frutos da pinha (Annona squamosa L.). Revista Brasileira de Fruticultura, v. 29, n. 2, p. 287-291, 2007.

CHAVAN, M.J; SHINDE, D.B.; NIRMAL, S.A. Major volatile constituents of Annona squamosa L. bark. Natural Product Research, v.8, p. 754-757, 2006.

CHEN, Y.; CAO, Y.; LI, F.; ZHU, X.; PENG, C.; LU, J.; CHEN, J.; LI, X.; CHEN, Y. Studies on anti-hepatoma activity of Annona squamosa L. pericarp extract. Bioorganic \& Medicinal Chemistry Letters, v. 27, n. 9, p.1907-1910, 2017.

CORDEIRO, M.C.R.; PINTO, A.C.Q.; RAMOS, V.H.V. O cultivo da pinha, fruta do conde ou ata no Brasil. Circular técnico - Embrapa Cerrado, n.9, p. 1-52, 2000.

EMBRAPA AGROINDÚSTRIA TROPICAL. Instruções técnicas para o cultivo da ateira. Fortaleza, n.1, 1998.

GUPTA, R.K.; KESARI, A.N.; MURTHY, P.S.; CHANDRA, R.; TANDON, V.; WATAL, G. Hypoglycemic and antidiabetic effect of ethanolic extract of leaves of Annona squamosa L. in experimental animals. Journal of ethnopharmacology, v. 99, n. 1, p. 75-81, 2005.

MEDINA, R.B.; SILVA, S.R.; RODRIGUES, K.F.D.; AVLÉS, T.C.; KAVATI, R. Produção de mudas de anonáceas. Coopercitrus, v.350, 2015.

MOHD, M.; ALAM, K.S.; MOHD, A.; ABHISHEK, M.; AFTAB, A. Antidiabetic activity of the aqueous extract of Annona squamosa in streptozotocin induced hyperglycemic rats. The Pharma Research, v. 2, p. 59-63, 2009.

NAIR, R.; AGRAWAL, V. A Review on the Nutritional Quality and Medicinal Value of Custard Apple-An Under Utilised Crop of Madhya Pradesh, India. International Journal of Current Microbiology and Applied Sciences, v. 6, n. 9, p. 1126-1132, 2017. 
NANDHAKUMAR, E.; INDUMATHI, P. In vitro antioxidant activities of methanol and aqueous extract of Annona squamosa (L.) fruit pulp. Journal of Acupuncture and Meridian Studies, v. 6, n. 3, p. 142-148, 2013.

PANDEY, N.; BARVE, M.D. Antioxidant activity of ethanolic extract of Annona squamosa Linn bark. International Journal of Pharmacy and Biological Sciences, v. 2, p. 16921697, 2011.

PARDHASARADHI, B.V.V.; REDDY, M.; ALI, A.M.; KUMARI, A.L.; KHAR, A. Differential cytotoxic effects of Annona squamosa seed extracts on human tumour cell lines: role of reactive oxygen species and glutathione. Journal of Bioscience and Bioengineering, $v$. 30, p. 237-244, 2005.

PARDHASARADHI, B.V.V.; REDDY, M.; ALI, A.M.; KUMARI, A.L.; KHAR, A. Antitumour activity of Annona squamosa seed extracts is through the generation of free radicals and induction of apoptosis. Indian Journal of Biochemistry and Biophysics, v. 41, p. 167172, 2004.

RAHMAN, M. M.; PARVIN, S.; HAQUE, M. E.; EKRAMUL, I. M.; MOSADIKK, M. A. Antimicrobial and cytotoxic constituents from the seeds of Annona squamosa. Fitoterapia, e.76, p. 484-489, 2005.

REN, Y.; ZHU, Z.; SUN, H.; CHEN, L. Structural characterization and inhibition on $\alpha-$ glucosidase activity of acidic polysaccharide from Annona squamosa, L. Carbohydrate Polymers, v. 174, p. 1-14, 2017.

SHIRWAIKAR, A.; RAJENDRAN, K.; KUMAR, C.D.; BODLA, R. Antidiabetic activity of aqueous leaf extract of Annona squamosa in streptozotocin-nicotinamide type 2 diabetic rats. Journal of ethnopharmacology, v. 91, n. 1, p. 171-175, 2004.

SUNANDA, P.; ANAND, K. Possible amelioration of hyperthyroidism by the leaf extract of Annona squamosa. Current Science, v.84, p. 1402-1404, 2003.

TU, W.S.; ZHU, J.H.; BI, S.X.; CHEN, D.H.; SONG, L.Y.; WANG, L.S.; ZI, J.C.; YU, R.M. 
Isolation, characterization and bioactivities of a new polysaccharide from Annona squamosa and its sulfated derivative. Carbohydrate Polymers, v. 152, p. 287-296, 2016.

VIANA, J. S. Implantação de um Pomar de Pinha irrigado no Distrito Federal. Boletim Técnico UPIS. Brasília, DF. 2005. 37p. 